This item was submitted to Loughborough's Institutional Repository (https://dspace.lboro.ac.uk/) by the author and is made available under the following Creative Commons Licence conditions.

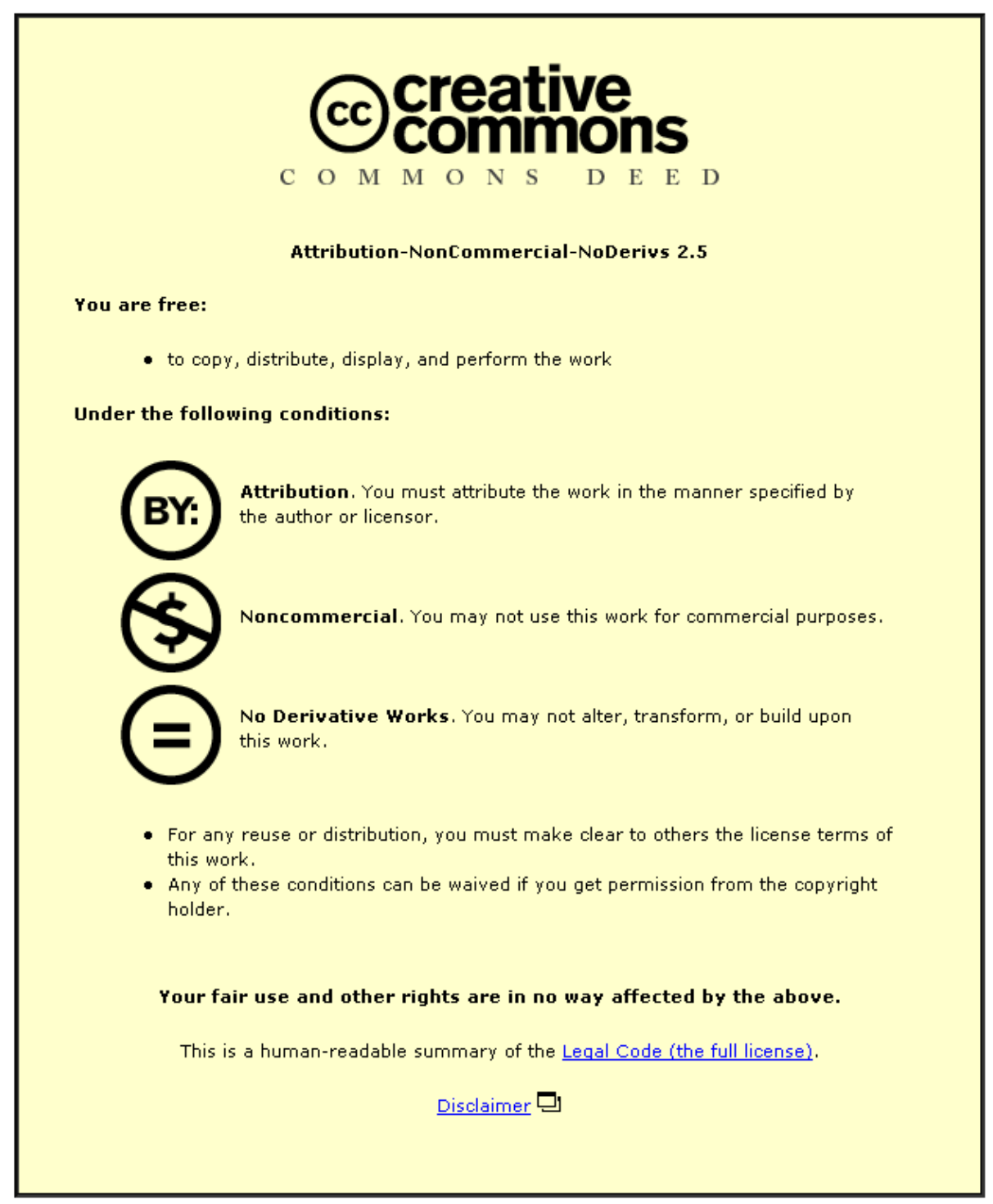

For the full text of this licence, please go to: http://creativecommons.org/licenses/by-nc-nd/2.5/ 


\title{
Innovation for Personalization: A Healthcare Case Study
}

\author{
Ron Summers SMIEEE, Sijung Hu MIEEE, Angelos Echiadis MIEEE, Vince Azorin-Peris and \\ Vassilios Chouliaras MIEEE.
}

\begin{abstract}
This paper describes a research and innovation platform for the development of ideas relating to the investigation of blood perfusion in peripheral tissue. The Loughborough Innovation Platform for Health Technologies (LIPHT) can be used to demonstrate the use of the research and innovation pipe-line in more than one dimension. For this paper the first dimension considered is that of 'blue sky' idea through to their exploitation for the benefit of users and at the same time creating a wealth stream; the second dimension is the changing market as the ideas develop - from a hospitalbased instrument operated by clinicians through to a point and click device for the use by the knowledgeable layman in the community. The starting point for these developments is a medical device known to many people as the 'finger clip' that measures arterial blood oxygen saturation; the end point is an optical device capable of imaging blood perfusion.
\end{abstract}

\section{INTRODUCTION}

$\mathrm{T}$ HE innovations for personalization of healthcare described in this paper trace the development of ideas in two separate research threads: the use of photoplethysmography (PPG) to provide information about blood perfusion; and multiscale representation of physiological detail to investigate processes at different levels of hierarchy simultaneously. Although research in each area has at least a ten year history, until two years ago they were being investigated in different parts of Loughborough University independently. Now brought together under the concept of the Loughborough Innovation Platform for Health Technologies (LIPHT) the research teams have joined their efforts. As will be demonstrated the innovations also have two dimensions: the first is the transformation of 'blue sky' ideas to a product; the second is the technological change from a hospital-based measurement system to a monitoring system useful for use in the home. Throughout, the research has been clinically driven, and there are a number of clinical applications - use cases - that

Manuscript received April 7, 2009.

All five authors are from the Department of Electronic and Electrical Engineering, Loughborough University, Loughborough, UK.

Prof. Ron Summers (corresponding author) is Professor of Information Engineering and can be reached via phone on +441509 635713; and e-mail at R.Summers@1boro.ac.uk

Dr. Sijung $\mathrm{Hu}$ is a Senior Research Fellow and can be reached via e-mail at $\underline{\text { S.Hu@lboro.ac.uk }}$

Dr. Angelos Echiadis is a Research Assistant and can be reached via email at A.Echiadis@1boro.ac.uk

Vince Azorin-Peris is a Research Student and can be reached via e-mail at V.Azorin-Peris@1boro.ac.uk

Vassilios Chouliaras is a Senior Lecturer and can be reached via e-mail at V.A.Chouliaras@,lboro.ac.uk can be highlighted. An example of a use case to demonstrate product development is the clinical measurement of oxygen delivery to the tissues - for this information about venous perfusion as well as arterial perfusion needs to be known. In terms of home-based measurements, the use of PPG in the information feedback loop for therapeutic oxygen delivery can also be considered a use case.

\section{RESEARCH AND INNOVATION PIPE-LINE}

\section{A. Outline Clinical Context}

Photoplethysmography (PPG) is a bio-monitoring technique that measures arterial pulsations in vivo based on optical properties of the interaction of light with matter. An attractive feature of the technique is that it provides real-time signals that are non-invasive, safe and painless. The underpinning measurement technology is centered on the assumption that changes in reflected (or transmitted) light intensity are as a consequence of changes in blood volume. In fact the changes in blood volume provides the dynamic pulse form that is reproducible with respect to time and provides a characteristic waveform. Differences in the waveform provide clinicians with diagnostic information although it must be remembered that the waveform changes its shape and amplitude as the arterial pulse travels further from the heart.

\section{B. University-based Research and Innovation}

A general model of the research and innovation pipe-line adopted within UK universities can be described as a voyage from the 'blue sky' ideas, through development of that idea into a research prototype. Typically, this first component of the model is funded through research grant awards from what can be diverse funding sources - UK Research Councils (for biomedical applications notably the Engineering and Physical Sciences Research Council, the Medical Research Council, and the BBSRC), direct from UK Government Departments (e.g. the Department of Health); a number of Trusts may have designated calls for proposals (e.g. The Wellcome Trust) and the many medical or health-related charities. From an international perspective, the EU Framework Programme is an attractive option, and there are often joint calls for proposals between the UK/EU and USA. A recent challenge to research proposers is the change in funding model adopted by the UK Government and its Research Councils, to one that is termed full economic costs. An unintended consequence of this change is that as charities and some Trusts have not adopted this 
model, a two tier funding system is in operation. 'High quality' research has become a misnomer that represents the origin of the funding rather than an indicator of what has been achieved.

There has been a perceptible shift in Universities in the UK to market not only the expertise of their academic staff, but also the findings of research. So, the second component of the pipe-line model is the development of the research prototype which requires the management of innovation and entrepreneurship. Common with other UK universities, Loughborough has seen an increase in the staff and activity of the Intellectual Property Office. Funding for this component can be more difficult as the number of available sources is very much less than the research component, and what is available is usually obtained via third party involvement. Although in general terms the staff of the Intellectual Property Office provide value, they may not have the same cultural involvement with the research to be marketed as those more directly involved.

A further way in which to engage with the Intellectual Property Office of a UK university is to form a spin-out company where the researchers and the University (through a chosen representative) begin a co-operative partnership. This route is good for first-time entrepreneurs (an oxymoron?) to obtain funding to further develop an idea or prototype.

\section{Research Landmarks}

A time-line of research landmarks is shown in Table I opposite, and provides relevant doctoral-level research work that has contributed to the development of the fusion of PPG technologies of the biophotonics team with multiscale modeling of the health informatics team.

In 1995 Pierre Jamali completed his $\mathrm{PhD}$ on using an innovative software architecture to encode the processes involved in intelligent patient monitoring. The so called 'multigraph' software architecture was the result of work at Vanderbilt University under the direction of Professor Janos Sztipanovits. Whereas it was developed as a consequence of their work on the NASA space laboratory, it provided the first inkling on what was to become the first architecture for multiscale models. Fusion of this architecture with Beer's viable system model meant that the hierarchical representations of the multigraph architecture were replaced with recursive ones to give a more faithful representation of physiology.

Matthew Hayes work on artifact reduction in the PPG signal, completed in 1998, acted as the precursor to future effort in biophotonics measurement technology. Two threads become identifiable: how to reduce movement artifact by investigating a non-contact method for data collection; and looking at oxygen delivery to the tissues. The former is the subject of Sharon Cheang's PhD thesis, completed in 2008. For oxygen delivery to the tissues, information from the venous side as well as the arterial side is required. It can be taken as a given that conventional PPG is appropriate for the measurement of arterial oxygen saturation. Work by Daniel
TABLE I

RESEARCH LANDMARKS

\begin{tabular}{|c|c|c|}
\hline Year & $\begin{array}{l}\text { BIOPHOTONICS } \\
\text { TEAM }\end{array}$ & $\begin{array}{c}\text { HEALTH INFORMATICS } \\
\text { TEAM }\end{array}$ \\
\hline 1995 & & $\begin{array}{l}\text { Multigraph Software } \\
\text { Architecture for Intelligent } \\
\text { Patient Monitoring [1] }\end{array}$ \\
\hline $\begin{array}{l}1996 \\
1997\end{array}$ & & \\
\hline 1998 & $\begin{array}{l}\text { PPG Artefact } \\
\text { Reduction [2] }\end{array}$ & \\
\hline $\begin{array}{l}1999 \\
2000 \\
2001\end{array}$ & & \\
\hline 2002 & $\begin{array}{l}\text { Non-invasive Venous } \\
\text { Oximetry [3] }\end{array}$ & \\
\hline 2003 & $\begin{array}{l}\text { Non-invasive Vascular } \\
\text { Assessment using PPG } \\
\text { [4] }\end{array}$ & \\
\hline $\begin{array}{l}2004 \\
2005\end{array}$ & & \\
\hline 2006 & & $\begin{array}{l}\text { Information Management } \\
\text { Applied to Bioinformatics [5] }\end{array}$ \\
\hline 2007 & & \\
\hline 2008 & $\begin{array}{l}\text { Evaluation of Venous } \\
\text { Oximetry [6] }\end{array}$ & $\begin{array}{l}\text { A Systemic Perspective of the } \\
\text { Bioinformatics Work Domain } \\
\text { [8] }\end{array}$ \\
\hline 2009 & $\begin{array}{l}\text { Non-contact PPG [7] } \\
\text { Opto-physiological } \\
\text { Modeling of Pulse } \\
\text { Oximetry [9] }\end{array}$ & \\
\hline
\end{tabular}

Chan completed in 2002 and Vincent Crabtree a year later provided the techniques for venous oximetry. As a worldfirst, these ideas provided the basis for forming a spin-out company that remains in operation today. Evaluation of venous oximetry was the subject of Angelos Echiadis' $\mathrm{PhD}$ thesis that was completed in 2008.

Not shown in Table I are the uses that the multigraph architecture were put in conventional patient monitoring. However, the ideas surfaced again in the work of Hiten Vyas in his $\mathrm{PhD}$ thesis that was completed in 2006 on an information management approach to managing data in bioinformatics applications. Here, one issue was the integration of data from an number of sources (i.e. databases) that are situated in many parts of the world. As well as non-homogenous data sources, the data themselves represented physiological processes at different spatial scales, and thus provided an impetus to look at multiscale physiology in more detail. Realizing that a systems approach to multiscale systems might bring rewards, Roliana Ibrahim investigated bioinformatics workflows in a $\mathrm{PhD}$ that was completed in 2008. The research landmarks are completed with the publication of the PhD thesis of Vicente AzorinPeris in 2009. This thesis provides an indication of the direction of future work, in that it describes the optophysiological modeling of pulse oximetry. 


\section{LIPHT: INNOVATIVE STEPS}

The following two methods will be used as exemplars to the innovations uncovered in LIPHT: venous oximetry; and PPG imaging.

The research components that covered venous oximetry resulted in a product - called 'Venox'. The Venox technologies constitute a novel and unique method for the non-invasive measurement of venous oxygen saturation $\left(\mathrm{SvO}_{2}\right)$. The Venox system can provide enhanced monitoring of cardiovascular function and as such has wide implications for clinical practice, health promotion, and human performance assessment. No similar technology exists for monitoring the oxygen levels in the venous system and hence the consumption and/or demand for oxygen by the tissues. Existing pulse oximeters can only measure arterial oxygen saturation due to the fact that their principle of operation relies on the presence of arterial pulsations. Venous blood flow does not present any pulsations, and as a consequence conventional pulse oximetry can not detect venous oxygen saturation. By introducing a series of pulsations of known low frequency via a finger sphygmomanometer, a pulsatile venous flow can be induced. By applying similar techniques to conventional pulse oximetry, the Venox system can distinguish between arterial and venous components of the pulsatile blood flow, and therefore it is possible to measure venous oxygen saturation. As both arterial oxygen saturation (input to tissues) and venous oxygen saturation (output from tissues) are now known, the tissue oxygen consumption can be derived. A patent for the Venox system was filed in March 2003 (PCT WO03/063697). A technology assessment of Venox has been carried out by comparing its results with the current 'gold' standard, the heart-lung bypass machine, on patients undergoing cardio-thoracic surgery. Statistical data analysis has confirmed that there is a strong correlation between venous oxygen saturation measured by both methods. A new Venox prototype is under development with an enhanced user interface design that uses touch screen technology for fast and simple operation.

The proposed PPG imaging system utilizes the expertise of bio-photonics, electronic systems design, and multiscale physiological modeling. The research extends the PPG portfolio indicated in Table I by investigating the real-time imaging of blood perfusion in tissues. In-vitro and in-vivo measurements of blood perfusion with oxygen supply and demand characteristics over an extended region of tissue will effectively enable scientists and clinicians to investigate the processes that are responsible for the transfer of oxygen from blood to tissue cells. Currently it is not possible to measure the oxygen content of veins and arteries simultaneously and independently, and thus it is not possible to measure the consumption of oxygen in a given tissue region. Key to achieving this functionality is the use of a multi-time gating approach to deliver light of different wavelengths and intensities via non-contact PPG. The reflected light comes from different depths of tissue from which a 3-D optical tomogram can be constructed. For success, advanced VLSI-based platforms designed with novel tool flows are required. Their use results in substantially reduced software development times while being 'correct-by-construction'. The proposed step change in computational speed will be enabled by the design of a dedicated photon transport engine using a System-on-Chip VLSI architecture. The third component of the PPG imaging technology is an adaptive physiological model representation of the images obtained that comprise visual physiological information at various levels of scale. These representations yield a powerful clinical diagnotic tool based on a case-based reasoning approach. Whereas image processing and storage will be uneventful, care will also be given to beyond storage processes such as digital preservation and archiving. This PPG imaging system will measure and access vascular blood perfusion, and display the tissue oxygen consumption mapping over an extended tissue area - without the need for direct skin contact or introducing any agent into the tissue. The proposed technology is non-invasive, safe and painless. A systems engineering methodology has been employed to describe the pre-clinical use cases. The first use case is hospital-based, building on existing research interests in monitoring cardiac output and managing heart failure, which can rapidly become life threatening. The condition is characterized by hypermetabolism (increased oxygen consumption, increased carbon dioxide production and impaired oxygen delivery). A second use case that is closely associated with research interests of the international community is circulation assessment in patients with diabetes. This is a disease syndrome that can manifest so-called 'diabetic foot', This condition is an example of a peripheral vascular disease that can quickly lead to ulcers and gangrene. The treatment for the latter is often amputation of the lower leg. It is anticipated that early changes in the microcirculation of the foot can be picked up by the PPG imaging system, and appropriate treatment given to prevent further morbidity. Other use cases will be explored, with specific choice depending on clinical need and opportunity.

\section{DISCUSSION}

The research strands that have come together to provide an integrated approach to PPG imaging have been introduced, and component technologies seen in Figure I below. It can be argued that the application of the integrated PPG imaging system has a data mining and knowledge discovery component via the use of the multiscale physiological model. Use cases have been indicated that demonstrate the transformation of hospital device to one that can be used in a closed or open loop control system in the home where decisions are required as to when to provide therapeutic intervention. 


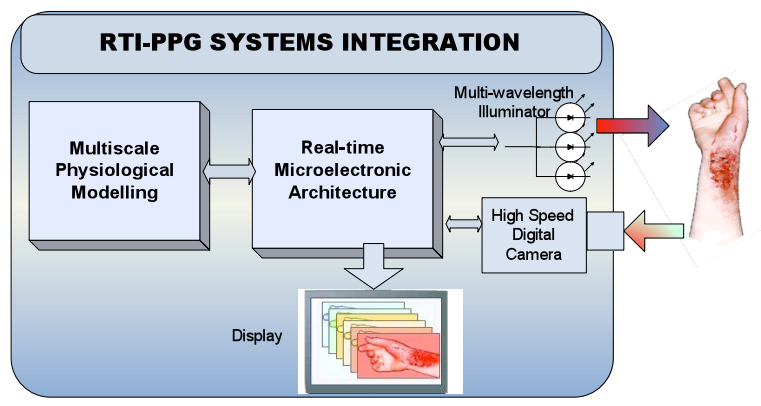

Figure 1: System Integration

Real-time assessment of blood perfusion is crucial to understand the progress of disease, and give an indication of the efficiency of the cardiovascular system. The PPG-based imaging system provides a real-time diagnostic capability and assessment for use in hospitals, medical and biomedical research centers, and for clinical trials of novel pharmaceuticals. It also has the possibility to make an impact within healthcare community settings, for example, via use in mobile screening facilities and use for personalized healthcare in the home. The imaging technology is significantly cost effective for microcirculation assessment but, more importantly, will encourage wider and more frequent testing of blood perfusion in peripheral tissues by clinicians and the general public.

Further use cases to explore include:

Vascular disease and circulatory function: In chronic venous incompetence (CVI), transcutaneous oxygen pressure and the density of capillaries in the ankle are often reduced. The evaluation of CVI through oxygen consumption imaging can become a simple, accurate and invivo assessment for clinicians to manage their intervention procedures. An applied example of such vascular disease is in some patients who have diabetes, where 'diabetic foot' is a condition that needs to be monitored aggressively, and any imaging system that can provide an early warning of CVI would be useful.

Cerebral blood perfusion: An opportunity has presented to use the PPG imaging system as a means of real-time assessment of cerebral blood perfusion. An existing international collaboration with Shanghai Jiao Tong University in China are partners in this research effort.

In vivo and in vitro microcirculation assessment: The PPG imaging system can assess microcirculation, erythema and pigmentation and could be a useful tool for oxygen consumption monitoring in burn patients.

Monitoring of hypoxia during exercise tests: Localized mapping of oxygen consumption before, during and after exercise tests give essential information for assessing ischemia, e.g. in brain ischemia, heart muscle ischemia and limb ischemia. The imaging system can also assess hypoxia induced in athletic performance testing, including effects of exposure to extreme altitudes and environments.
With an ability to provide rapid and effective images of peripheral tissues, the methods adopted may transfer to mainstream clinical diagnostics. Such a focus can be seen as a method to reduce risk in many use cases, such as 'diabetic foot' referred to earlier in the context of the diabetic patient. Thus, this innovative imaging modality has a great potential to impact on the quality of life of the general population. Should the device in production be within the planned relatively low cost range, PPG imaging technologies has the added bonus of being able to be afforded in third world countries where diseases of peripheral vasculature are common.

\section{CONCLUSION}

This paper has presented an outline summary of the work of two research groups within Loughborough University over a period of time that extends back to the 1990s. As well as demonstrating the dynamics of the work with respect to the research and innovation pipe-line, an indication of how the research has moved towards the opportunity for personalized healthcare is afforded. There are also other healthcare use cases not referred to (e.g. monitoring of sepsis in neonates) and non-healthcare applications such as in homeland security where bio-identification using noncontact reflection photoplethysmography is a possibility.

\section{ACKNOWLEDGMENT}

All of the authors of this paper acknowledge the work of our colleague Professor Peter Smith to whom this paper is dedicated. Without him we would still be working in independent research teams, and his untimely death has robbed us of a true visionary.

\section{REFERENCES}

[1] P. Jamali, "Application of the multigrapgh software architecture to intelligent patient monitoring", PhD Thesis, Department of Systems Science, City University, 1995.

[2] M. Hayes, "Artefact reduction in Photoplethysmography", $\mathrm{PhD}$ Thesis, Department of Electronic and Electrical Engineering, Loughborough University, 1998.

[3] D. F-C. Chan, "Non-invasive venous oximetry through venous blood volume modulation", PhD Thesis, Department of Electronic and Electrical Engineering, Loughborough University, 2002.

[4] V. Crabtree, "Non-invasive vascular assessment using photoplethysmography", PhD Thesis, Department of Electronic and Electrical Engineering, Loughborough University, 2003.

[5] H. Vyas, "Information management applied to bioinformatics", $\mathrm{PhD}$ Thesis, Department of Information Science, Loughborough University, 2006.

[6] A. A. Echiadis, "Development and evaluation of venous oximetry", $\mathrm{PhD}$ Thesis, Department of Electronic and Electrical Engineering, Loughborough University, 2008.

[7] S. P. Y. Cheang, "Feasibility of non-contact photoplethysmography", $\mathrm{PhD}$ Thesis, Department of Electronic and Electrical Engineering, Loughborough University, 2008.

[8] R. Ibrahim, "A systemic perspective of the bioinformatics work domain", PhD Thesis, Department of Information Science, Loughborough University, 2008.

[9] V. Azorin-Peris, "Optophysiological modeling of pulse oximetry", $\mathrm{PhD}$ Thesis, Department of Electronic and Electrical Engineering, Loughborough University, 2009. 